\title{
Revisión sistemática sobre modelos multidimensionales del bienestar y su medición en estudiantes universitarios
}

\author{
Rubia C. Cobo-Rendón(1), María V. Pérez-Villalobos ${ }^{(2)}$, Alejandro E. Díaz-Mujica(2) y Diego J. García-Álvarez ${ }^{(3)}$ \\ (1) Facultad de Ciencias Sociales, Programa de Doctorado en Psicología, Universidad de Concepción, \\ Concepción-Chile. (correo-e: rubiacobo@udec.cl) \\ (2) Facultad de Ciencias Sociales, Dpto. de Psicología, Universidad de Concepción, Concepción-Chile. \\ (correo-e: marperez@udec.cl; adiazm@udec.cl) \\ (3) Facultad de Ciencias Políticas Administrativas y Sociales, Escuela de Psicología, Universidad Rafael Urdaneta, \\ Maracaibo-Venezuela. (correo-e: diegogarcia_123@hotmail.com)
}

Recibido Ago. 8, 2019; Aceptado Sep. 29, 2019; Versión final Oct. 30, 2019, Publicado Abr. 2020

\begin{abstract}
Resumen
Los objetivos de este estudio fueron caracterizar las investigaciones publicadas en la aliteratura internacional sobre modelos multidimensionales del bienestar en estudiantes universitarios e identificar los instrumentos de medición empleados en este contexto. Se analizaron trabajos publicados en Web of Science ${ }^{\mathrm{TM}}$, Scopus ${ }^{\circ}$ y SciELO ${ }^{\circledR}$ desde 1990 a 2019, los descriptores fueron estudiantes universitarios, bienestar y florecimiento en inglés y español. Se identificaron 550 publicaciones de las cuales 42 fueron seleccionadas para formar parte de la muestra. Según el diseño, dos estudios fueron de tipo cualitativo (3.03\%), cuatro de tipo experimental (3.03\%), diez de tipo instrumental (30.30\%) y 25 de tipo empírico asociativo (63.63\%). Se identificaron cuatro instrumentos de medición. El estudio del bienestar multidimensional es relevante el contexto de la educación superior por su vinculación con variables de salud mental y por los beneficios que ofrece en el éxito en la universidad. Se concluye que se necesita desarrollar más investigaciones sobre la conceptualización y medición del bienestar multidimensional en estudiantes universitarios, en especial en contextos latinoamericanos.
\end{abstract}

\section{Systematic review in multidimensional models of well-being and its measurement in university students}

\begin{abstract}
The objectives of this study were to characterize the research published in the international literature on multidimensional models of well-being in university students and to identify the measuring instruments used in this context. Papers published in Web of Science ${ }^{\mathrm{TM}}$, Scopus ${ }^{\circledR}$ and SciELO ${ }^{\circledR}$ from 1990 to 2019 were analyzed, the descriptors were university students, well-being and flourishing, in English and Spanish. 550 publications were identified, out of which 42 were selected to be part of the sample of this study. According to the design, two studies were of qualitative type (3.03\%), four of experimental type (3.03\%), ten of instrumental type (30.30\%) and 25 of empirical associative type (63.63\%). Four measuring instruments were identified. The study of multidimensional well-being is relevant to the context of higher education because of its link with mental health variables and because of the benefits it offers in success in university. More research is needed on the conceptualization and measurement of multidimensional well-being in university students, especially in latin american contexts.
\end{abstract}

Keywords: systematic review; psychological well-being; subjective well-being; university students; higher education 


\section{INTRODUCCIÓN}

La forma de comprender y estudiar el bienestar ha tenido un gran avance, existe un considerable aumento de su estudio en diversos contextos (Castro-Solano, 2009). Desde sus inicios el bienestar ha sido estudiado por medio de dos tradiciones filosóficas: la tradición eudaimónica (bienestar psicológico), vinculada al desarrollo del potencial humano y la tradición hedónica (bienestar subjetivo), referida a la satisfacción con la vida y la experimentación de emociones positivas. Diversos autores han llegado al conceso que el bienestar es un constructo multidimensional, siendo el concepto de florecimiento la presencia de altos niveles de bienestar en ambas tradiciones (Diener et al., 2010; Hone et al., 2014; Huppert et al., 2009; Keyes, 2002; Seligman, 2011). Desde esta perspectiva se asume que el bienestar eudaimónico y hedónico son constructos conceptuales independientes, pero al mismo tiempo están correlacionados fuerte y positivamente, sin embargo, no lo suficiente para comprometer la validez en su medición (Hone et al., 2014; Seligman, 2011; Waterman, 2008).

El florecimiento es considerado como un fenómeno intrínsecamente valioso y es descrito como la actualización continua y dinámica del potencial humano (Wolbert et al., 2015). Hasta la fecha ha sido definido desde cuatro enfoques teóricos principales. El primero concibe el florecimiento como la presencia de síntomas hedónicos y el funcionamiento positivo organizados en un continuum de salud mental (Keyes, 2002); El segundo, como la combinación de sentirse bien y funcionar eficazmente, por medio de la presencia de características positivas (estabilidad emocional, vitalidad, optimismo, resiliencia, autoestima) y características de funcionamiento positivo (compromiso, competencia, significado) (Huppert et al., 2009), El tercero, como el alcance de emociones positivas, compromiso, relaciones positivas, una vida con propósito y logros, conocido como PERMA por sus siglas en inglés (Seligman, 2011) y cuarto, se conceptualiza al florecimiento por la presencia en las personas de competencia, autoaceptacion, vida con significado, relaciones saludables, optimismo y logro (Diener et al., 2010).

El estudio del bienestar multidimensional en los últimos años ha proporcionado resultados relevantes; investigaciones independientes reportaron que adolescentes florecientes presentaron menores dificultades en ajuste psicológico, así como también menor prevalencia de síntomas depresivos y mayor presencia de comportamientos prosociales (Singh et al., 2015). Por el contrario, adolescentes con menor florecimiento presentaron mayores conductas de riesgo para su salud (Venning et al., 2013). Adultos florecientes presentan altos niveles de concientización, extroversión y bajos niveles de neuroticismo (Schotanus-Dijkstra, et al., 2016a), adicionalmente presentan menos riesgo de padecer trastornos del estado de ánimo y de ansiedad (Schotanus-Dijkstra et al., 2016b). Hasta la fecha se han presentado revisiones sistemáticas sobre el florecimiento y su medición en niños y en adolescentes (Witten et al., 2019; Rose et al., 2017), sin embargo, poco se conoce sobre el aporte de esta temática en el contexto de estudiantes universitarios.

La justificación de este trabajo reside en el aporte para la investigación futura sobre bienestar multidimensional en la Educación Superior. Es relevante el estudio del florecimiento en el contexto estudiantil universitario debido a que el bienestar desde la perspectiva eudaimónica se basa en la noción de una vida que valga la pena vivir enmarcada en compromisos personales con valor intrínseco, metas ajustadas y logros a lo largo del ciclo vital, y es allí donde los estudios universitarios pueden cobrar un valor significativo en el bienestar de una persona, pensado en que los mismos estén enfocados en un proyecto de vida que permitan las vías al florecimiento. Al mismo tiempo, la noción actual del bienestar incluye en su comprensión el impacto de las emociones positivas, que obedece a la tradición hedónica del mismo, que se encuentra mucho menos reportada en la literatura científica y administrativa e incluso confundido con el término de satisfacción de los estudiantes hacia la universidad (Elwick y Cannizzaro, 2017). El estudio del florecimiento está recibiendo mayor atención, en el contexto universitario pudiese proveer de grandes oportunidades a los estudiantes para desarrollarse como educandos, futuros trabajadores y ciudadanos (Howell y Buro, 2015), con la idea de que la universidad pueda facilitar seriamente espacios dirigidos a promover el disfrute por la vida estudiantil universitaria, enseñanza de habilidades socio-emocionales, promoción de las fortalezas del carácter y resiliencia que favorezca a los estudiantes en el camino de su formación profesional e igualmente a su crecimiento personal.

En este sentido, el desarrollo de revisiones sistemáticas ofrece la posibilidad de informar y actualizar a los investigadores y población general a partir de la integración objetiva de la información disponible (PeresteloPérez, 2013). Esta metodología provee de información para el avance científico de un tema de interés, permite la identificación de las conclusiones y hallazgos relevantes para poder identificar caminos posibles a seguir en la investigación (Sánchez -Meca, 2010). Este estudio plantea una revisión sistemática sobre el bienestar multidimensional en estudiantes universitarios, teniendo en cuenta el poco consenso que existe en la literatura científica e incluso en documentos de administración educativa universitaria sobre lo que realmente se entiende por florecimiento en el contexto estudiantil universitario (Elwick y Cannizzaro, 2017). De ahí que esta revisión sistemática busca dar respuesta a las preguntas: ¿Qué tipo de investigaciones sobre bienestar multidimensional se han desarrollado en estudiantes universitarios y cuáles son los instrumentos de medición 
empleados en ese contexto? En tal sentido, los objetivos de este trabajo son: 1) Caracterizar las investigaciones sobre modelos multidimensionales del bienestar en estudiantes universitarios y 2) Identificar los instrumentos de medición empleados en este contexto.

\section{METODOLOGIA}

El presente trabajo es una revisión sistemática cualitativa, puesto que no se aplicarán técnicas de análisis estadístico para integrar y sistematizar los efectos de los resultados reportados en las investigaciones seleccionadas (Sánchez-Meca, 2010), se consideraron los lineamientos de la declaración PRISMA para revisiones sistemáticas (Moher et al., 2009). A continuación, se describen cada una de las etapas planteadas según esta metodología y en la figura 1 se representa el proceso para la selección de los artículos de la revisión.

\section{Identificación}

Esta etapa consistió en la búsqueda de estudios sobre modelos multidimensionales del bienestar en estudiantes universitarios. Se consultaron artículos de investigación publicados desde 1990 hasta 10 julio del 2019, considerando este marco temporal debido a que las primeras conceptualizaciones sobre bienestar multidimensional fueron presentadas a inicio de los años noventa (Witten, et al., 2019). Se emplearon los buscadores científicos de la Web of Science ${ }^{\mathrm{TM}}$, Scopus ${ }^{\circledR}$ y SciELO ${ }^{\circledR}$, empleándose los descriptores en inglés y en español de "estudiantes universitarios" AND "bienestar" OR "florecimiento". Se empleó el software de gestión bibliográfica online Refworks para la organización de los trabajos identificados en la búsqueda. En total fueron identificados 550 trabajos en las bases de datos seleccionadas.

\section{Selección}

Los criterios de selección de los trabajos fueron: 1) artículos presentados con diseño cualitativo o cuantitativo, 2) publicados en inglés o español, 3) que estudiaran al bienestar desde la visión hedónica y eudaimónica en conjunto, 4) desarrollados en el contexto universitario. Los criterios de exclusión fueron 1) documentos como revisiones, conferencias, capítulos de libros o cartas al editor, 2) textos no disponibles, 3) investigaciones que estudiaran y/o midieran solo un aspecto del bienestar, 4) investigaciones de un contexto distinto al de estudiantes universitarios (docentes, pacientes, trabajadores, adultos mayores, población general) e 5) investigaciones que fueran en un nivel educativo distinto al universitario (estudiantes de jardín de infantes, primaria, secundaria, estudiantes de postgrado/doctorado).

\section{Extracción y análisis de la información}

Luego de la identificación de los trabajos, eliminación de duplicados y del acceso completo a los documentos, esta etapa consistió la revisión de los trabajos, para esto tres revisores realizaron el proceso de lectura de los artículos para su selección, primero en conjunto realizaron una primera lectura de títulos y resúmenes para confirmar la congruencia de los descriptores, en este proceso fueron eliminados 126 trabajos. Después de forma individual los revisores realizaron la lectura completa de los trabajos restantes considerando los criterios de inclusión y exclusión previamente establecidos. Con todos los trabajos seleccionados, los revisores crearon tablas de resumen con datos de cada trabajo.

Las tablas generadas fueron comparadas por el cuarto revisor para corroborar la consistencia en los resultados obtenidos. En los casos donde no existía claridad en la inclusión de algún trabajo, se solicitó la valoración del cuarto revisor para comparar resultados y llegar a un acuerdo para el reporte final. La calidad metodológica de los artículos seleccionados se realizó de la siguiente manera: para los estudios instrumentales se tomaron en cuenta la descripción de la delimitación conceptual, la información sobre la construcción, adaptación y evaluación de las propiedades psicométricas (Carretero-Dios y Pérez, 2007), para los estudios cuantitativos y cualitativos se analizaron la coherencia presente entre el objetivo del estudio, el método empleado y el análisis de los resultados realizado.

\section{RESULTADOS}

Fueron seleccionados un total de 42 artículos teniendo en cuenta los criterios establecidos. Para responder al objetivo de caracterizar las investigaciones sobre modelos multidimensionales del bienestar en estudiantes universitarios en la tabla 1 se describen aspectos específicos como país de los participantes, tamaño de la muestra, tipo de diseño empleados, técnica de recolección de información utilizada y principales hallazgos de cada estudio. Para responder al objetivo de identificar los instrumentos de medición empleados en este contexto, luego del registro de las escalas en la tabla 1, se realizó el análisis de las propiedades psicométricas según el reporte de los estudios seleccionados. En la tabla 2 se describen estos resultados según el país en donde fueron desarrollados cada trabajo. 


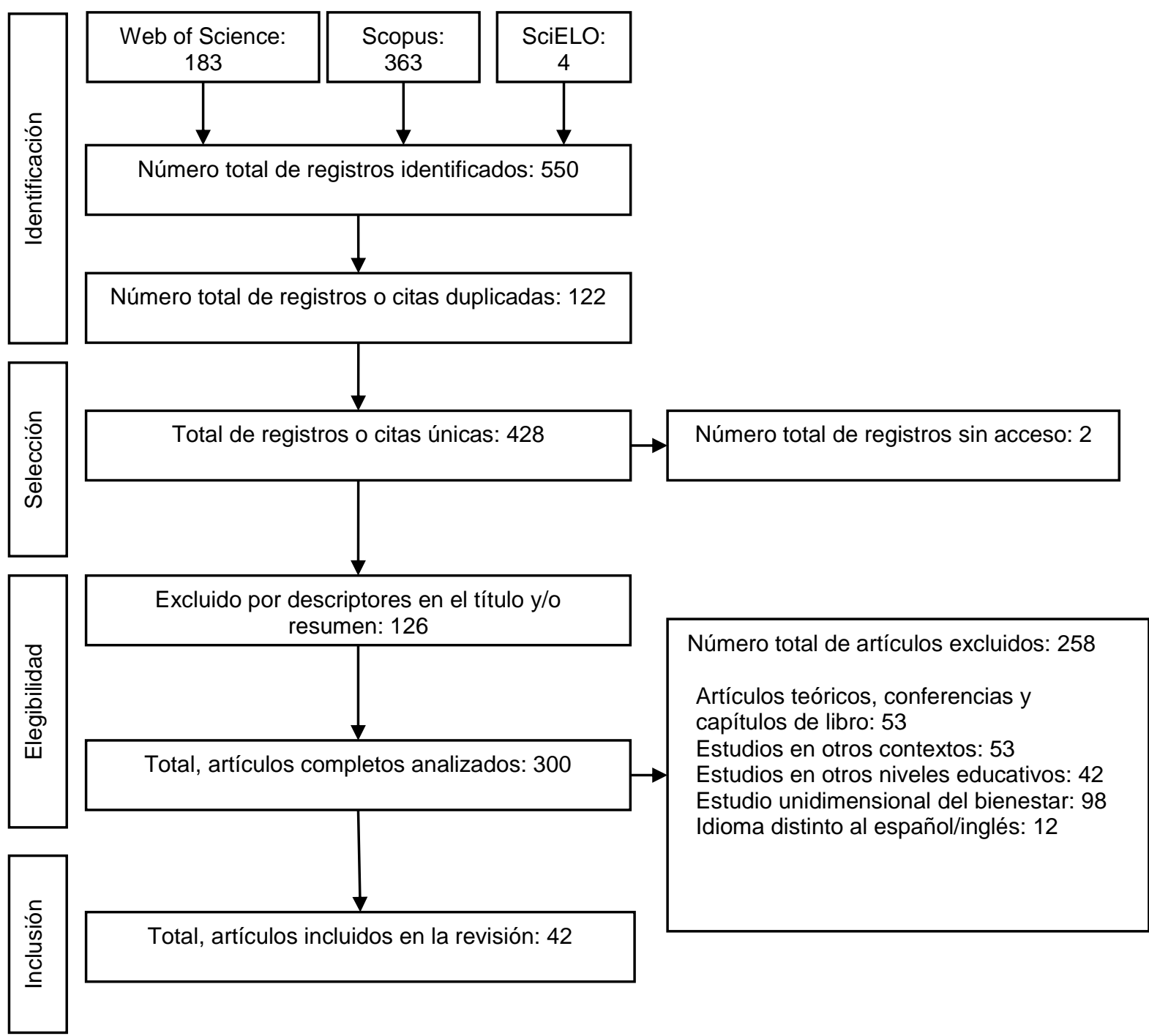

Fig. 1: Fases de la revisión sistemática.

\section{Modelos multidimensionales del bienestar en estudiantes universitarios.}

El estudio del florecimiento en el contexto universitario se está desarrollando en distintas partes del mundo. Los países con mayor producción de investigación fueron Estados Unidos ( $n=9 ; 21.42 \%)$, Turquía ( $n=7$; $16.66 \%)$, España $(n=3 ; 7.14 \%)$, Sudáfrica $(n=3 ; 7.14 \%)$, Filipinas $(n=2 ; 4.76 \%)$ y Croacia $(n=2 ; 4.76 \%)$. Se identificaron tres estudios transculturales (Pozo-Muñoz et al., 2016; Vorone et al., 2012; Lambert et al., 2019) y dos estudios desarrollados con muestras latinoamericanas (Cassaretto y Martínez, 2017; Pozo-Muñoz et al., 2016). Respecto al tamaño de la muestra, los trabajos con diseño cualitativo presentaron investigaciones con menos de 100 participantes. En el caso de las investigaciones cuantitativas, la cantidad de participantes se agrupó mayormente entre 201 y 300 sujetos ( $\mathrm{N}=10 ; 23.80 \%)$ y el otro rango de mayor proporción fue de 501-1000 participantes $(\mathrm{N}=10 ; 23.80 \%)$. Los resultados presentan el reporte de respuestas total de 25.853 estudiantes universitarios. En cuanto al tipo de investigación empleado, dos estudios fueron de tipo cualitativo (4.73\%), diez de tipo instrumental $(23.80 \%)$, cuatro de tipo experimental $(9.52 \%)$, y 26 de tipo empírico asociativo (61.90\%) (Ato et al., 2013). Las técnicas de recolección de información empleadas, en los estudios cualitativos fue la entrevista y en los estudios cuantitativos, se emplearon varias escalas de medición.

En relación a los principales hallazgos de las investigaciones, los resultados de los estudios cualitativos indicaron que el florecimiento es esencial en el proceso de adaptación de los estudiantes a la vida universitaria (Knoesen y Naudé, 2018) y que las teorías sobre bienestar multidimensional son útiles para fomentar el crecimiento personal de los estudiantes (D'raven et al., 2015). Los resultados de los estudios empíricos asociativos, indican una relación positiva del florecimiento con variables como la satisfacción con la vida (Fong y Loi, 2016; Satici y Uysal, 2015), la felicidad subjetiva (Satici y Uysal, 2015), las necesidades psicológicas básicas (Mesurado et al., 2016; Miljković y Jurčec, 2016) y el uso de estrategias de regulacion emocional adaptativas (Basson y Rothmann, 2018). Los estudios reportaron relaciones positivas con variables vinculadas al desarrollo óptimo como la atención plena (Akin y Akin, 2015b; Duan, 2016) y las fortalezas intelectuales e interpersonales (Duan, 2016). 


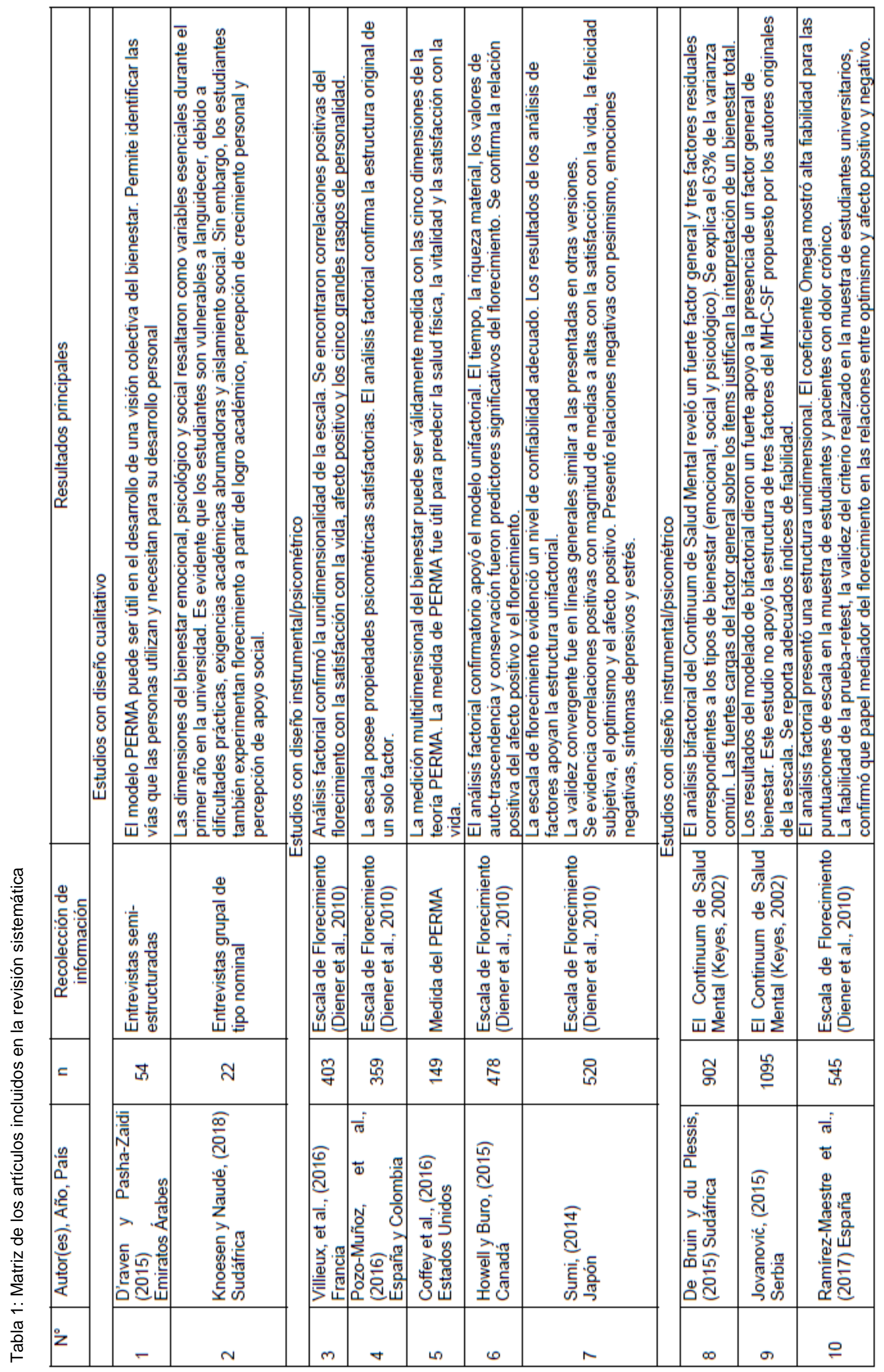




\begin{tabular}{|c|c|c|c|c|c|c|c|c|c|c|}
\hline 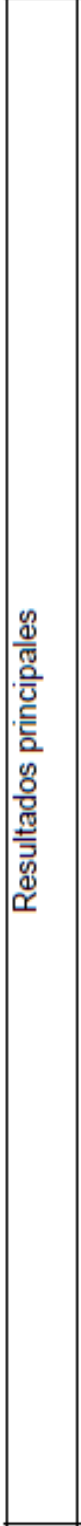 & 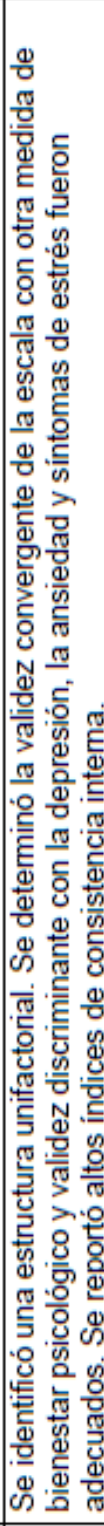 & 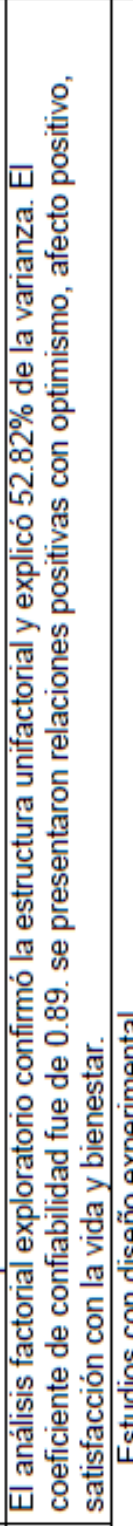 & 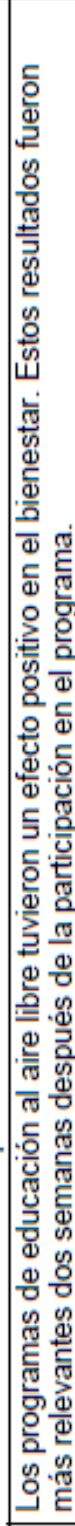 & 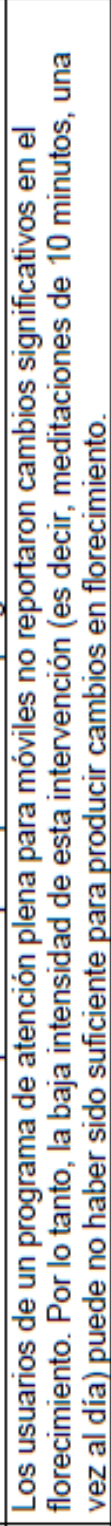 & 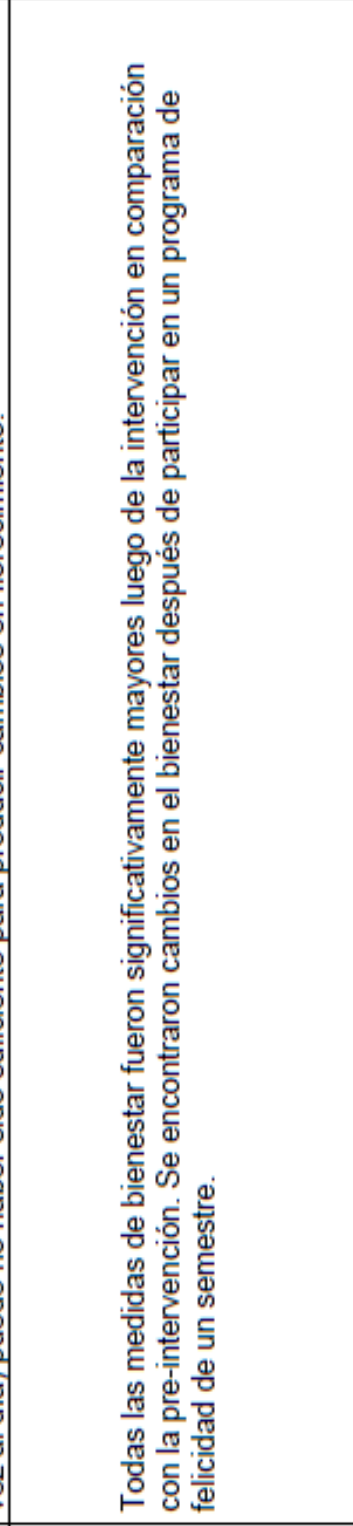 & 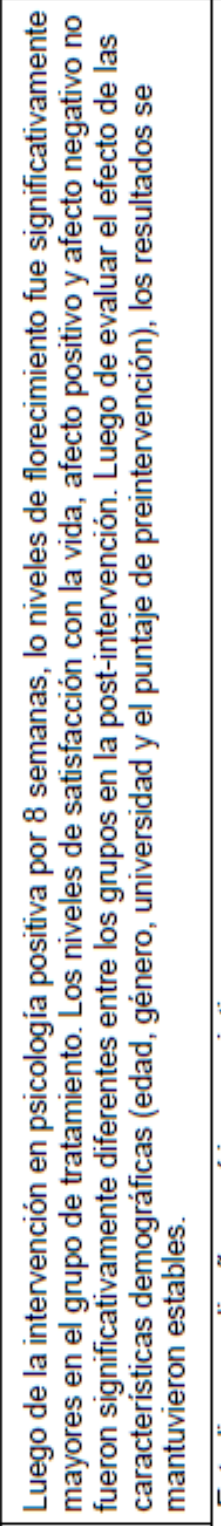 & 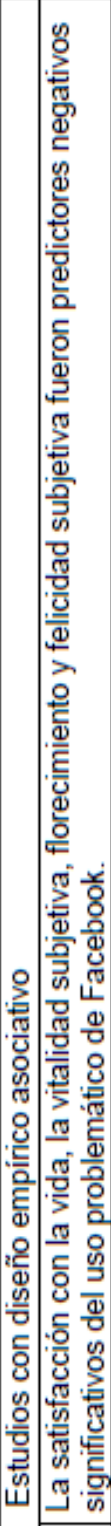 & 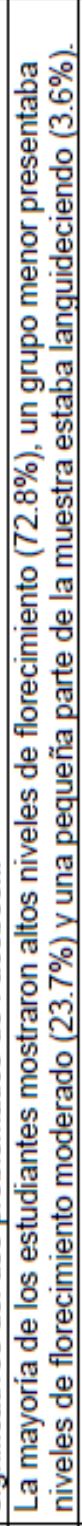 & 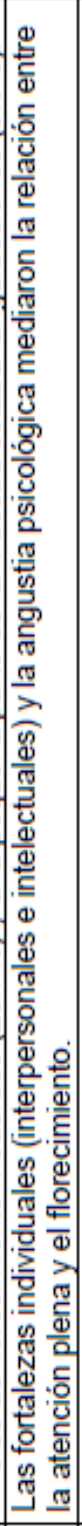 & 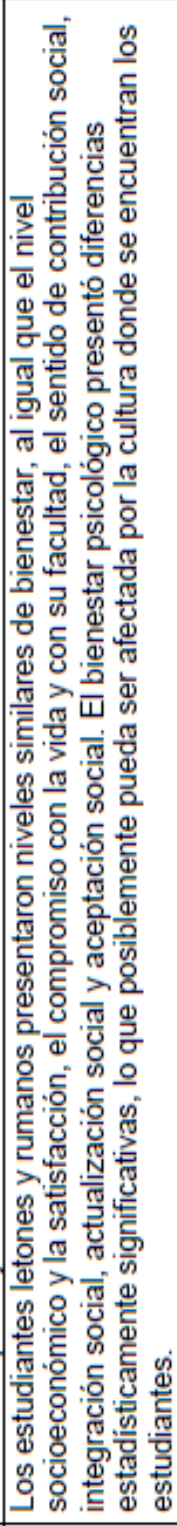 \\
\hline 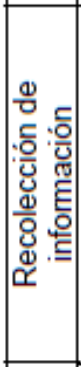 & 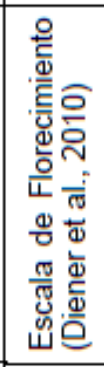 & 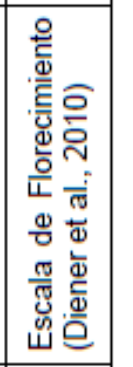 & 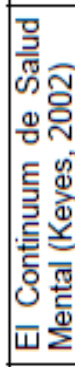 & 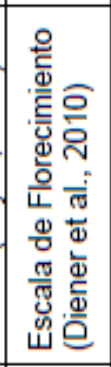 & 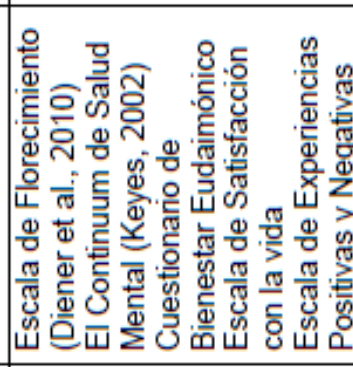 & 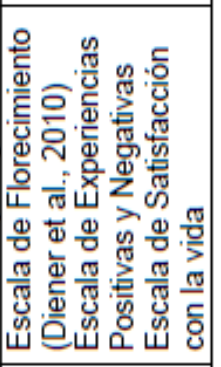 & 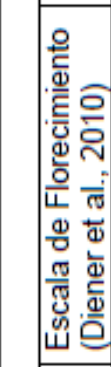 & 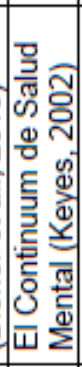 & 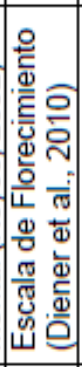 & 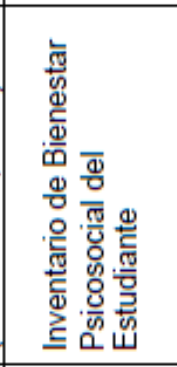 \\
\hline c & ్ㅗㅇ & బ్ & 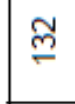 & 号 & $\ddot{\ddot{~}}$ & $\sqrt{\mathrm{m}}$ & $\bar{m}$ & i & ஓ & क \\
\hline 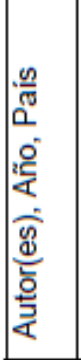 & 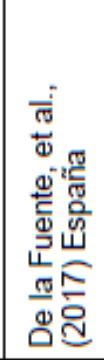 & 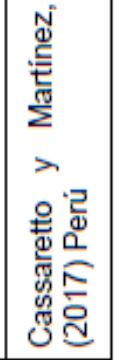 & 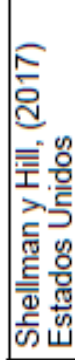 & 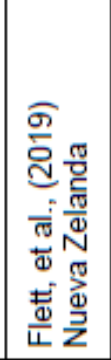 & 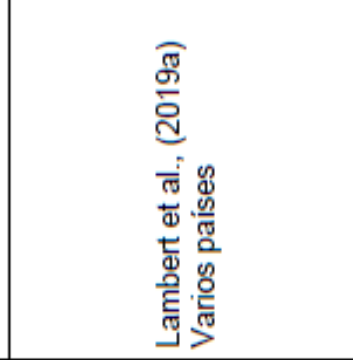 & 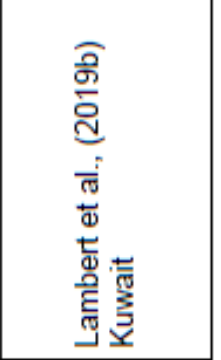 & 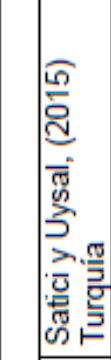 & 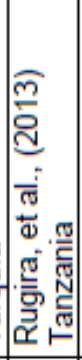 & 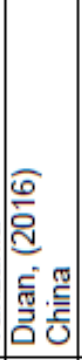 & 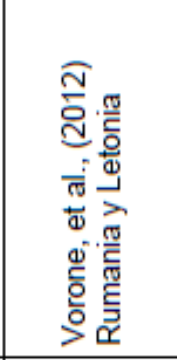 \\
\hline z & $=$ & $\stackrel{\sim}{\simeq}$ & $\stackrel{m}{-}$ & $\underset{\square}{ \pm}$ & $\stackrel{\circ}{\sim}$ & 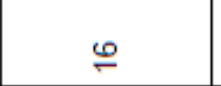 & 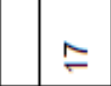 & $\stackrel{\infty}{-}$ & $\stackrel{\mathscr{P}}{\leftarrow}$ & 이 \\
\hline
\end{tabular}




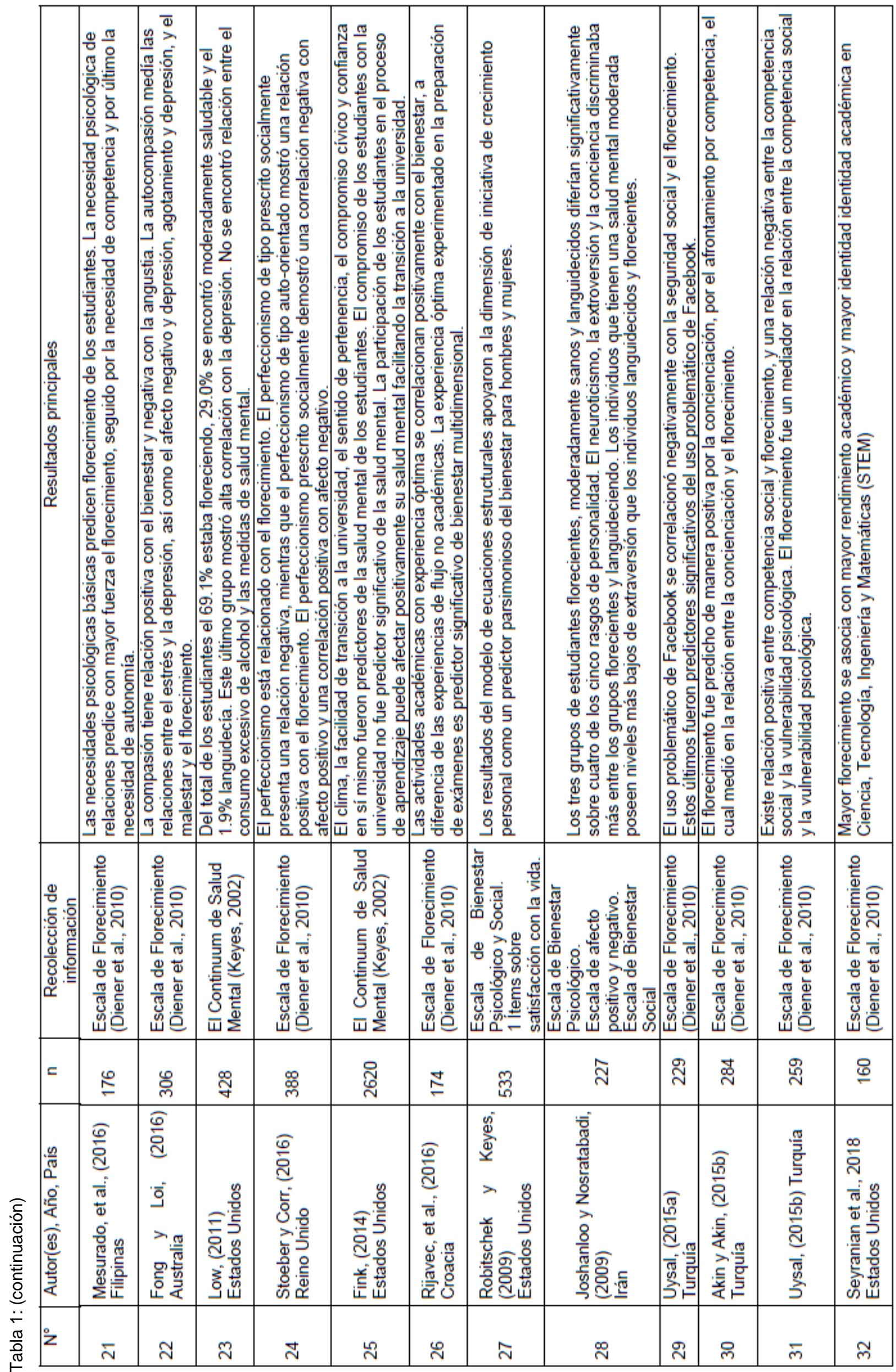




\begin{tabular}{|c|c|c|c|c|c|c|c|c|c|c|}
\hline 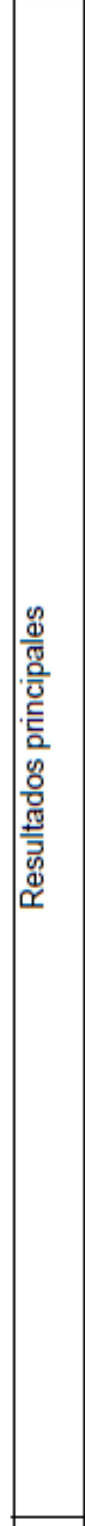 & 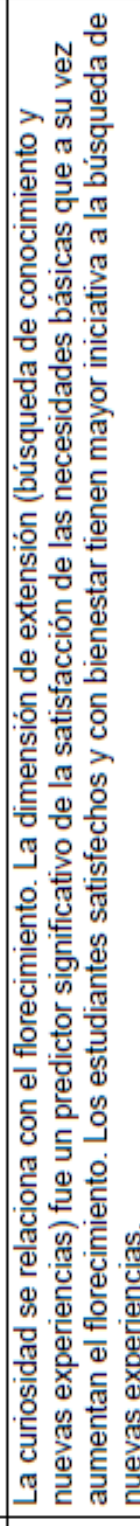 & 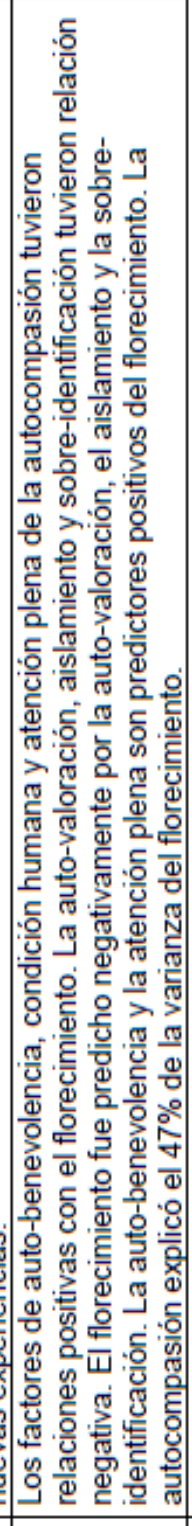 & 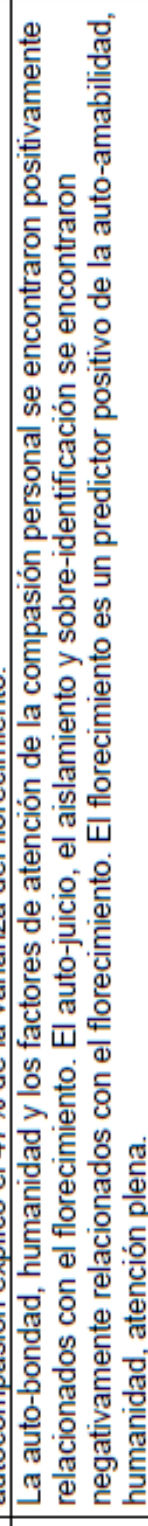 & 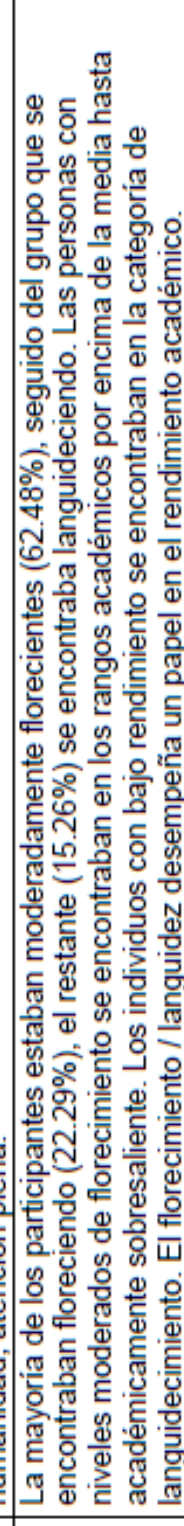 & 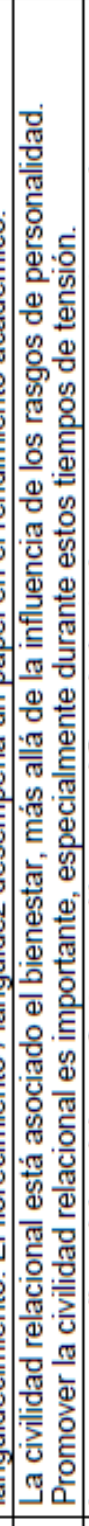 & 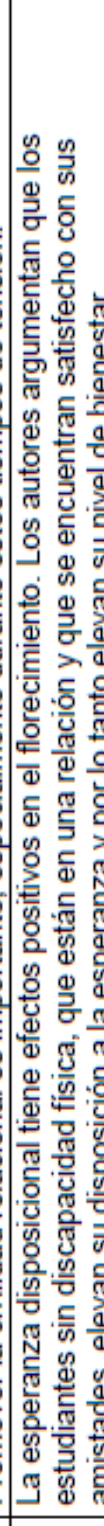 & 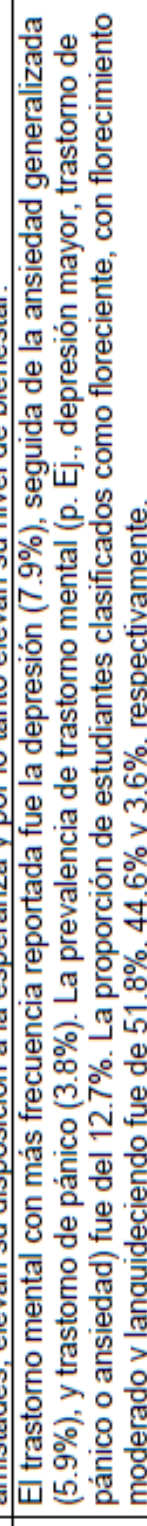 & 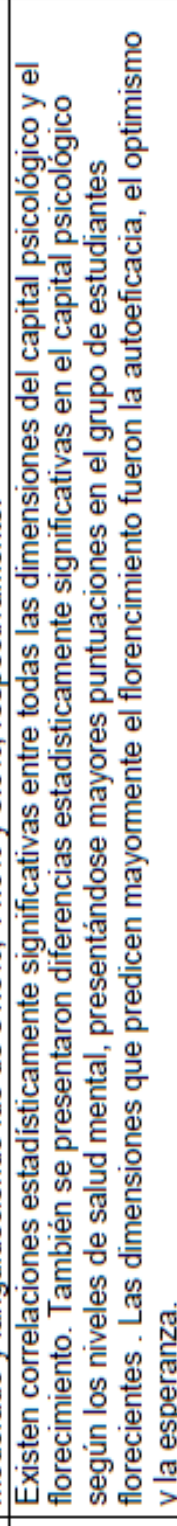 & 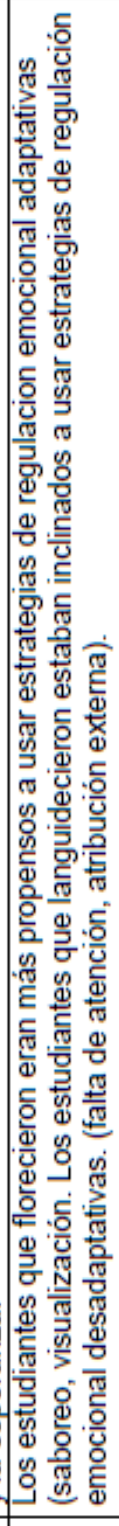 & 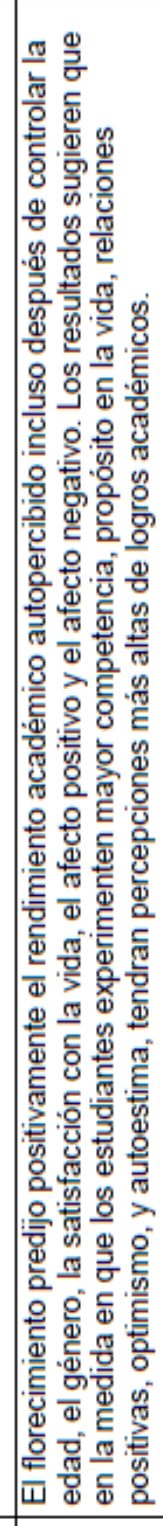 \\
\hline 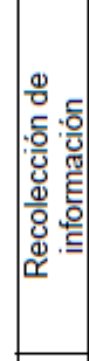 & 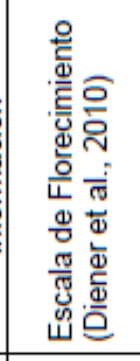 & 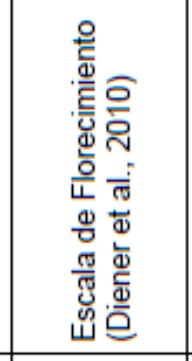 & 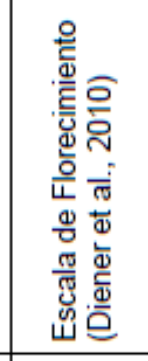 & 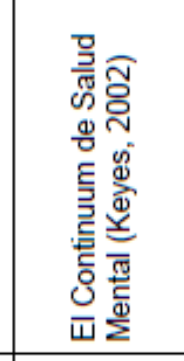 & 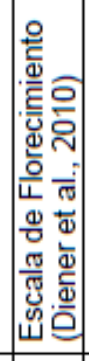 & 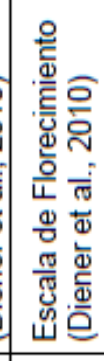 & 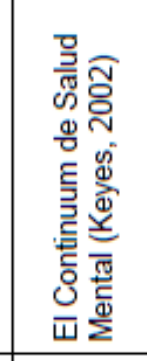 & 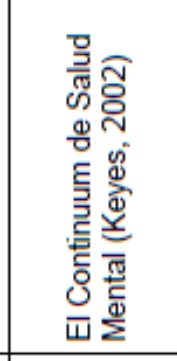 & 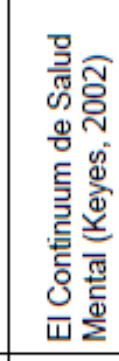 & 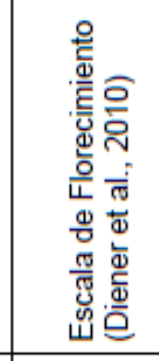 \\
\hline$=$ & $\stackrel{\infty}{m}$ & $\stackrel{\infty}{\underset{\sim}{*}}$ & 岱 & $\infty$ & $\stackrel{?}{\sim}$ & $\bar{\infty}$ & & & & \\
\hline 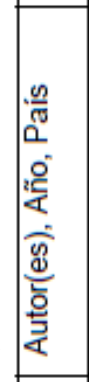 & 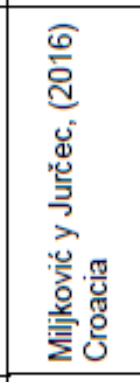 & 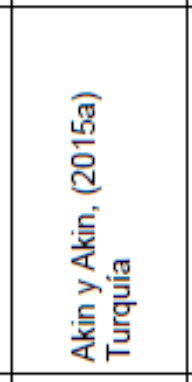 & 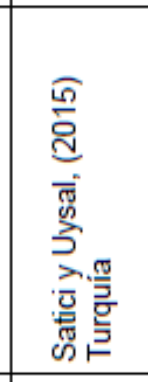 & 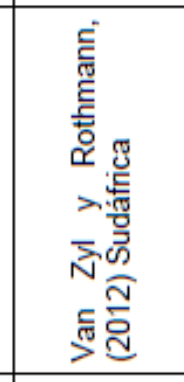 & 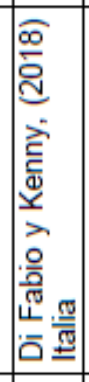 & 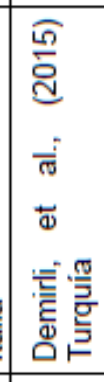 & 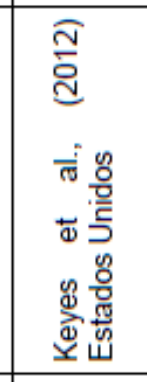 & 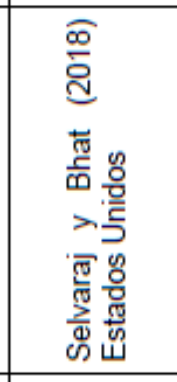 & 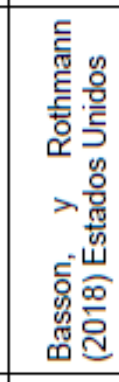 & 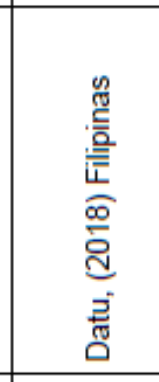 \\
\hline$z$ & & লे & 卢 & $\stackrel{m}{ }$ & $\hat{m}$ & & m & 워 & & Fั \\
\hline
\end{tabular}


Se presentan relaciones positivas con el afrontamiento por competencia (Akin y Akin, 2015b) y rasgos de personalidad como la extroversión, alta conciencia y amabilidad (Joshanloo y Nosratabadi, 2009). En el caso de variables orientadas al trabajo académico el bienestar multidimensional mostró relaciones positivas con la vitalidad (Coffey et al., 2016; Satici y Uysal, 2015), la iniciativa (Robitschek y Keyes, 2009), la curiosidad (Miljković y Jurčec, 2016), el capital psicológico (Selvaraj y Bhat, 2018) y la experiencia óptima (Rijavec, Ljubin-Golub y Olčar, 2016). Otros estudios reportaron relaciones positivas con variables de beneficio social como la autocompasión (Fong y Loi, 2016; Satici et al., 2013; Akin y Akin, 2015a), la competencia social (Uysal, 2015b) y las conductas cívicas (Di Fabio y Kenny, 2018). El bienestar multidimensional desempeña un papel positivo en el rendimiento académico (Van Zyl y Rothmann, 2012; Datu, 2018; Seyranian et al., 2018) y en la identidad académica en Ciencia, Tecnología, Ingeniería y Matemáticas (Seyranian et al., 2018) es una variable mediadora en la relación entre la competencia social y la vulnerabilidad psicológica en estudiantes universitarios (Uysal, 2015b). El bienestar multidimensional se asoció negativamente con depresión (Fong y Loi, 2016; Low, 2011), afecto negativo (Miljković y Jurčec, 2016; Stoeber y Corr, 2016), angustia, estrés y burnout (Fong y Loi, 2016) y es un predictor negativo para el uso problemático de Facebook (Akin y Akin, 2015b; Satici y Uysal, 2015).

Adicionalmente, las variables como atención plena, iniciativa, preparación para exámenes, necesidades psicológicas básicas, esperanza disposiciones, búsqueda de conocimiento, autoeficacia y optimismo fueron identificados como predictores positivos del florecimiento (Akin y Akin, 2015b; Demirli et al., 2015; Mesurado et al., 2016; Miljković y Jurčec, 2016; Robitschek y Keyes, 2009; Satici et al., 2013; Selvaraj y Bhat, 2018). Los análisis descriptivos mostraron que la mayoría de los estudiantes se encontraban floreciendo (Keyes et al., 2012; Low, 2011; Rugira et al., 2013, Basson y Rothmann, 2018), solo un estudio reportó resultados donde la mayoría de los estudiantes estaban moderadamente florecientes $(n=149)$, seguidos de florecientes $(n=35)$ y por último en menor medida languideciente $(n=40)$ (Joshanloo y Nosratabadi, 2009). Con respecto a los trabajos con diseño experimental, los autores reportan que los programas de intervención en educación al aire libre tienen impacto a largo plazo en el florecimiento de los estudiantes (Shellman y Hill, 2017). Así mismo los programas de bienestar para estudiantes universitarios tienen un efecto positivo en su florecimiento (Lambert et al., 2019a; Lambert et al., 2019b), resultado distinto en programas online sobre atención plena donde no se encontraron efectos significativos (Flett et al., 2019).

\section{Instrumentos de medida empleados}

Los estudios empíricos y psicométricos analizados permitieron identificar varios instrumentos de medida para el estudio del florecimiento, la escala más utilizada fue la Escala de Florecimiento ( $n=26 ; 61.90 \%)$, seguido por el Continuum de Salud Mental $(n=11 ; 26.19 \%)$, Medida para el PERMA $(n=1 ; 2.38 \%)$ y por último el Inventario de Bienestar Psicosocial del Estudiante Universitario $(n=1 ; 2.38 \%)$. Cinco investigaciones (11.90\%) emplearon una batería de instrumentos para medir los dominios del bienestar de forma independiente (psicológico, social, subjetivo). Para analizar las características de los instrumentos de medida de modelos de bienestar multidimensional en estudiantes universitarios, en la tabla 2 se reportan los aspectos considerados para su análisis, siendo estos el país en los que se reportan estudios, el índice de consistencia interna y la estructura factorial confirmada.

Tabla 2. Características Psicométricas de los instrumentos de medida empleados.

\begin{tabular}{|c|c|c|c|}
\hline $\begin{array}{c}\text { Nombre del } \\
\text { instrumento/Autor(es)/Año }\end{array}$ & País & $\begin{array}{c}\text { Consistencia interna } \\
\text { (Alpha Crombach " } \alpha \text { " / Omega McDonalds " } \omega \text { ") }\end{array}$ & $\begin{array}{l}\text { Estructura } \\
\text { factorial }\end{array}$ \\
\hline \multicolumn{4}{|c|}{ Escala de Florecimiento (Diener et al., 2010) } \\
\hline $\begin{array}{l}\text { Akin y Akin, (2015a), (2015b); } \\
\text { Satici y Uysal, (2015); Uysal, } \\
\text { (2015a), (2015b); Demirli et al., } \\
(2015)\end{array}$ & Turquía & $\alpha \geq .72$ & No reportan \\
\hline $\begin{array}{l}\text { Cassaretto Bardales y Martínez } \\
\text { Uribe, (2017) }\end{array}$ & Perú & $\alpha=.89$ & Unidimensional \\
\hline Duan, (2016) & China & $\alpha=.87$ & No reportan \\
\hline Fong y Loi, (2016) & Australia & $\alpha=.91$ & No reportan \\
\hline Howell y Buro, (2015) & Canadá & $\alpha=.89$ & Unidimensional \\
\hline $\begin{array}{l}\text { Mesurado et al., (2016); Datu, } \\
(2018)\end{array}$ & Filipinas & $\alpha>=.87$ & No reportan \\
\hline $\begin{array}{l}\text { Miljković y Jurčec, (2016); } \\
\text { Rijavec et al., (2016) }\end{array}$ & Croacia & $\alpha \geq .80$ & No reportan \\
\hline Pozo-Muñoz et al., (2016) & Colombia & $\alpha=.88$ & Unidimensional \\
\hline $\begin{array}{l}\text { Pozo-Muñoz et al., (2016); } \\
\text { Ramírez-Maestre et al., (2017); } \\
\text { De la Fuente et al. (2017) }\end{array}$ & España & $\alpha=.81-.85$ & Unidimensional \\
\hline Stoeber y Corr (2016) & Reino Unido & $\alpha=.89$ & No reportan \\
\hline
\end{tabular}


Tabla 2 (continuación)

\begin{tabular}{|c|c|c|c|}
\hline $\begin{array}{l}\text { Nombre del } \\
\text { instrumento/Autor(es)/Año }\end{array}$ & País & $\begin{array}{c}\text { Consistencia interna } \\
\text { (Alpha Crombach " } \alpha \text { " / Omega McDonalds " } \omega \text { ") }\end{array}$ & Estructura factorial \\
\hline Sumi, (2014) & Japón & $\alpha=.95$ & Unidimensional \\
\hline Villieux et al., (2016) & Francia & $\alpha=.82$ & Unidimensional \\
\hline Flett, et al., (2019) & $\begin{array}{l}\text { Nueva } \\
\text { Zelanda }\end{array}$ & No reportan & No reportan \\
\hline Lambert et al., (2019a) & Varios países & $\alpha>.84$ & No reportan \\
\hline Lambert et al., (2019b) & Kuwait & $\alpha>.67$ & No reportan \\
\hline Di Fabio y Kenny, (2018) & Italia & $\alpha=.92$ & No reportan \\
\hline Seyranian et al., (2018) & $\begin{array}{l}\text { Estados } \\
\text { Unidos }\end{array}$ & No reportan & No reportan \\
\hline \multicolumn{4}{|c|}{ El Continuum de Salud Mental (Keyes, 2002) } \\
\hline $\begin{array}{l}\text { De Bruin y du Plessis, (2015); } \\
\text { Van zyl y Rothmann (2012) }\end{array}$ & Sudáfrica & No reporta & $\begin{array}{l}\text { Factor general y } \\
\text { tres } \\
\text { independientes }\end{array}$ \\
\hline Jovanović, (2015) & Serbia & $\begin{array}{c}\omega=.81 \text { General } \\
\omega>.10 \text { Subescalas }\end{array}$ & Un factor general \\
\hline $\begin{array}{l}\text { Keyes et al., (2012); Selvaraj y } \\
\text { Bhat (2018); Basson, y } \\
\text { Rothmann (2018); Shellman y } \\
\text { Hill (2017), Low (2011); Fink } \\
\text { (2014); }\end{array}$ & $\begin{array}{l}\text { Estados } \\
\text { Unidos }\end{array}$ & $\begin{array}{c}\alpha=.92 \text { General } \\
\alpha>.81 \text { Subescalas }\end{array}$ & No reportan \\
\hline Rugira et al., (2013) & Tanzania & $\alpha=.79$ General & No reportan \\
\hline Lambert et al., (2019) & Varios países & $\alpha=.85$ & No reportan \\
\hline \multicolumn{4}{|c|}{ Medida para el PERMA } \\
\hline Coffey et al., (2016) & $\begin{array}{l}\text { Estados } \\
\text { Unidos }\end{array}$ & $\begin{array}{c}\text { Emociones positivas: } \alpha=.79 \\
\text { Relaciones: } \alpha=.52 \\
\text { Compromiso: } \alpha=.66 \\
\text { Logros: } \alpha=.62\end{array}$ & $\begin{array}{l}\text { Estructura con } 5 \\
\text { factores. }\end{array}$ \\
\hline \multicolumn{4}{|c|}{ Inventario de Bienestar Psicosocial del Estudiante Universitario } \\
\hline \multirow[t]{2}{*}{ Vorone et al., (2012) } & Rumania & $\alpha \geq .72$ & No reportan \\
\hline & Letonia & $\alpha \geq .75$ & No reportan \\
\hline
\end{tabular}

\section{DISCUSIÓN}

El presente estudio se planteó como objetivos 1) Caracterizar las investigaciones sobre modelos multidimensionales del bienestar en estudiantes universitarios, e 2) Identificar los instrumentos de medición empleados en este contexto. Para ello se realizó una revisión sistemática de la literatura desde 1990 hasta 10 julio del 2019 en las bases de datos Web of Science, Scopus y SciELO.

\section{Características de las investigaciones sobre modelos multidimensionales}

El florecimiento o bienestar multidimensional es un concepto que se ha vuelto relevante en el contexto educativo, puesto que la educación puede impactar en la vida de las personas. La incorporación del fomento del florecimiento en este contexto ha mostrado que las instituciones educativas no solo se enfocan en propiciar el conocimiento académico sino también el desarrollo del potencial humano. Hallazgos relevantes encontrados en los estudios seleccionados concluyen que el florecimiento es un constructo que beneficia a la vida de los universitarios. A nivel de salud se exhiben relaciones negativas con la depresión, angustia, estrés y burnout, sintomatología que suele manifestarse en los jóvenes durante este periodo de sus vidas (Conley et al., 2014; Stallman, 2010). Existen beneficios en términos académicos de vinculación del florecimiento con variables como la vitalidad, iniciativa, curiosidad, sin embargo, aún no queda claro como este constructo podría impactar en aspectos propios de la vida universitaria como rendimiento académico real, adaptación, vocación, hábitos de estudio, procrastinación y abandono de los estudios. Esto hace relevante considerar el estudio del bienestar en aspectos propios del contexto universitario, permitiendo aportar de forma más precisa a las necesidades presentes en la educación universitaria.

Sobre el diseño empleado en los estudios analizados, se identificaron un número importante de estudios psicométricos en distintos países, esto demuestra la importancia en el desarrollo de medidas objetivas que permitan un acercamiento objetivo al fenómeno de estudio (Carretero-Dios y Pérez, 2007). La realización de adaptaciones de medidas del bienestar ya propuestas, como es el caso de la Escala de Florecimiento (Diener et al., 2010) y El Continuum de Salud Mental (Keyes, 2002), es relevante en la comprensión de este fenómeno en estudiantes de distintas latitudes y facilita la posible comparación del bienestar considerando los mismos marcos teóricos, disminuyendo las dificultades en la generalización de los aportes presentes en las investigaciones (Cooke et al., 2016; Jayawickreme et al., 2012). 
Desde el punto de vista operativo como fortaleza todos los estudios psicométricos describen con claridad los objetivos planteados. En algunos trabajos $(n=7)$ se presentan tamaños de muestra adecuados para la realización de los análisis estadísticos, en especial de los análisis factoriales (Lloret-Segura et al., 2014), se presentan adecuados índices de consistencia interna y se consideran los métodos estadísticos en base al tipo de escala empleado ya sea de tipo categórico o de intervalo (Streiner et al., 2015). Los procedimientos para la estimación de la validez son considerados en base a las propuestas teóricas e investigaciones previas, fortaleciendo la continuidad de los hallazgos encontrados en otros estudios de este tipo. Todos los estudios consideraron análisis factoriales como método principal para el análisis de validez, algunos de ellos complementaron estos resultados con análisis de validez discriminante y convergente. Sin embargo, no todos los estudios psicométricos describen la estrategia empleada para asegurar la equivalencia conceptual entre la versión original de los instrumentos y la versión traducida (Muñiz, et al., 2013). En la presente revisión también se presentan tres estudios transculturales, este hallazgo es una fortaleza puesto que permite explorar como los aspectos culturales intervienen en el florecimiento. El empleo de este tipo de estudios permitirá aumentar la comprensión de la naturaleza del bienestar y la generalización de sus resultados a distintas culturas.

En lo referente a la calidad metodológica de los estudios empíricos todos los estudios mostraron coherencia entre los objetivos planteados y los análisis efectuados, sin embargo, se presentaron inconsistencias con respecto a la definición del bienestar. La mayoría de las investigaciones conceptualizan el bienestar como estado de desarrollo personal óptimo en donde el individuo puede evidenciar el uso de su potencial, experimentar emociones positivas y evaluar su satisfacción con la vida, considerándolo como un constructo multidimensional, sin embargo, otros estudios definen al florecimiento como una dimensión o aspecto de la experiencia óptima (Low, 2011) o como un predictor del bienestar (Satici y Uysal, 2015). En tal sentido aún se presentan inconsistencias con respecto a la conceptualización de este constructo en términos de constituirse como un concepto que integra las visiones del bienestar.

\section{Instrumentos de medición empleados}

La presente revisión identificó cuatro instrumentos diseñados en base a tres de las cuatro perspectivas teóricas presentadas hasta la fecha. La escala de Florecimiento diseñada en base a la teoría de florecimiento propuesta por el mismo autor (Diener et al., 2010), presenta adecuados índices de consistencia interna en estudiantes de distintos países, se confirma su estructura factorial unidimensional y se presentan niveles satisfactorios de validez convergente con variables como satisfacción con la vida, afectos positivos (Villieux et al., 2016), optimismo y estrés percibido (Cassaretto y Martínez, 2017; Sumi, 2014). Del mismo modo, El Continuum de Salud Mental, escala basada en la teoría propuesta por Keyes (2002) ha mostrado adecuados niveles de consistencia interna y de validez en países como Estados Unidos, Sudáfrica y Tanzania. Sin embargo, no se ha mantenido la estructura factorial propuesta en los estudios seleccionados, lo resalta la necesidad de investigar sobre estos aspectos. La medida de PERMA, diseñada para la medición del modelo propuesto por Seligman (2011), mostró adecuados niveles de consistencia interna y finalmente, el inventario de Bienestar Psicosocial del estudiante Universitario es un instrumento diseñado en base a distintos planteamientos teóricos sobre bienestar subjetivo, psicológico y social en conjunto (Negovan, 2010). Presentó adecuados índices de consistencia interna. La validez del instrumento presentó de bajos a moderados coeficientes de correlación con variables como autoestima, felicidad subjetiva y satisfacción con la vida.

Los resultados encontrados en la presente revisión evidencian un incremento en las investigaciones sobre el bienestar en estudiantes universitarios, sin embargo, todavía queda mucho por desarrollar, se presentan en su mayoría estudios de tipo descriptivos enfocados a contextos específicos, se evidencia pocos estudios con participantes de países latinoamericanos. La presencia de estudios que evalúen el impacto del florecimiento en este contexto aún es incipiente. Es importante continuar el desarrollo de estudios de validación o adaptación de instrumentos en contextos latinoamericanos, en ese caso considerar la presentación y descripción detallada de la metodología empleada para asegurar la correcta equivalencia conceptual entre los ítems durante los procesos de traducción de las escalas. Se hace necesario diseñar y evaluar programas de intervención que permitan conocer el efecto del florecimiento en variables de relevancia para el contexto universitario, como el rendimiento académico, el aprendizaje profundo, la autorregulación del aprendizaje, la adaptación a la vida universitaria, entre otros.

La presente revisión fue desarrollada para proporcionar una visión más amplia de las investigaciones y medición sobre el bienestar multidimensional en estudiantes universitarios. Sobre las limitaciones se presenta la consideración de solo tres bases de datos de publicaciones científicas y el considerar artículos publicados en dos idiomas (inglés y español), aspectos que podrían generar sesgos no deseados en los resultados presentados. El estudio del florecimiento es relevante no solo en la escuela sino también en el contexto universitario, beneficiando la comprensión de este concepto en la vida de los jóvenes. Parece haber un interés 
creciente en el uso de instrumentos para la medición del bienestar con adecuados procesos de adaptación, validez y confiabilidad que permitan el avance en la comprensión de este constructo en los estudiantes de este nivel, sin embargo, es claro que aún queda mucho trabajo por hacer en referencia a su definición y medición.

\section{CONCLUSIONES}

De los resultados mostrados, su análisis y discusión se pueden obtener las siguientes conclusiones sobre las investigaciones de modelos multidimensionales del bienestar en estudiantes universitarios. 1) La mayoría de los estudios identificados tienen un diseño empírico asociativo, seguido de estudios de tipo psicométrico. 2) Las investigaciones se han enfocado en comprobar la relación del florecimiento con variables positivas, de impacto social y de salud mental. En menor medida se han estudiado la relación con variables académicas. 3) Los estudios en variables académicas indican que el florecimiento desempeña un papel positivo en el rendimiento y la identidad de los estudiantes. 3) La escala de Florecimiento es la más empleada para la investigación en este contexto. 4) La mayor cantidad de investigaciones identificadas fueron en estudiantes de Turquía y Estados Unidos. Se identificaron pocos estudios desarrollados en estudiantes universitarios latinoamericanos.

\section{AGRADECIMIENTOS}

Este trabajo se realizó como parte del Proyecto de investigación más amplio, CONICYT/FONDECYT 1161502, Modelo explicativo de la permanencia y el abandono de los estudios universitarios, basado en procesos cognitivo motivacionales. Agradecemos a CONICYT por su colaboración.

\section{REFERENCIAS}

Akin, A. y Akin, U., Examining the Predictive role of Self-compassion on Flourishing in Turkish University Students, doi: 10.6018/analesps.31.3.192041, Anales de Psicología, 31(3), 802-807 (2015a).

Akin, A. y Akin, A., Mediating role of Coping Competence on the Relationship Between Mindfulness and Flourishing, doi:10.1016/j.sumpsi.2015.05.005, Suma Psicológica, 22(1), 37-43 (2015b).

Ato, M, López J. y Benavente, A., Un sistema de Clasificación de los Diseños de Investigación en Psicología, doi: 10.6018/analesps.29.3.178511, Anales de Psicología, 29(3), 1038-1059 (2013).

Basson, M. J., y Rothmann, S., Flourishing: Positive Emotion Regulation Strategies of Pharmacy Students, doi: 10.1111/ijpp.12420, International Journal of Pharmacy Practice, 26(5), 458-464 (2018).

Carretero-Dios, H. y Pérez, C., Standards for the Development and Review of Instrumental studies: Considerations about test selection in Psychological Research , International Journal of Clinical and Health Psychology, ISSN: 1697-2600, 7(3), 863-882 (2007).

Cassaretto, M. y Martínez, P., Validación de las Escalas de Bienestar, de Florecimiento y Afectividad, Pensamiento Psicológico, ISSN: 1657-8961, 15 (1), 19-31 (2017).

Castro-Solano, A., El Bienestar Psicológico: cuatro décadas de progreso, Revista Interuniversitaria de Formación del Profesorado, ISSN: 0213-8646, 23(3), 43-72 (2009).

Coffey, J., Wray-Lake, L., Mashek, D. y Branand, B., A Multi-Study Examination of Well-Being Theory in College and Community Samples, doi: 10.1007/s10902-014-9590-8, Journal of Happiness Studies, 17(1), 187-211 (2016).

Conley, C., Kirsch, A., Dickson, D. y Bryant, F., Negotiating the Transition to College, doi:10.1177/2167696814521808, Emerging Adulthood, 2(3), 195-210 (2014).

Cooke, P., Melchert, T. y Connor, K., Measuring Well-being: A Review of Instruments, doi: 10.1177/0011000016633507, The Counseling Psychologist, 44(5), 730-757 (2016).

Datu, J. A. D. Flourishing is Associated with Higher Academic Achievement and Engagement in Filipino Undergraduate and High School Students, doi: 10.1007/s10902-016-9805-2, Journal of Happiness Studies, 19(1), 27-39 (2018).

De Bruin, G. y Du Plessis, G., Bifactor Analysis of El Continuum de Salud Mental (Keyes, 2002) (MHC-SF), doi: 10.2466/03.02.PR0.116k20w6, Psychological Reports, 116(2), 438-446 (2015).

De la Fuente, R., Parra, A. y Sánchez-Queija, I., Psychometric Properties of Escala de Florecimiento (Diener et al., 2010) and Measurement Invariance Between Two Samples of Spanish University Students, doi: 10.1177/0163278717703446, Evaluation y the Health Professions, 40(4), 409-424 (2017).

Demirli, A., Türkmen, M. y Arık, R., Investigation of Dispositional and State Hope Levels' Relations with Student Subjective Well-being, doi: 10.1007/s11205-014-0607-9, Social Indicators Research, 120(2), 601-613 (2015).

Di Fabio, A., y Kenny, M., Intrapreneurial Self-capital: A key Resource for Promoting Well-being in a Shifting Work Landscape, doi: 10.3390/su10093035, Sustainability, 10(9), 3035 (2018). 
Diener, E., Wirtz, D. y otros cinco autores, New Well-being Measures: Short Scales to Assess Flourishing and Positive and Negative Feelings, doi: 10.1007/s11205-009-9493-y, Social Indicators Research, 97(2), 143-156 (2010)

Duan, W., Mediation role of Individual Strengths in Dispositional Mindfulness and Mental Health, doi: 10.1016/j.paid.2016.04.078, Personality and Individual Differences, 99, 7-10 (2016).

Elwick, A. y Cannizzaro, S., Happiness in Higher Education, doi: 10.1111/hequ.12121, Higher Education Quarterly, 71(2), 204-219 (2017).

Fink, J.E., Flourishing: Exploring Predictors of Mental Health within the College Environment, doi: 10.1080/07448481.2014.917647, Journal of American College Health, 62(6), 380-388 (2014).

Flett, J. A., Hayne, H. y otros tres autores, Mobile Mindfulness Meditation: a Randomised Controlled Trial of the Effect of two popular Apps on Mental Health, doi: 10.1007/s12671-018-1050-9, Mindfulness, 10(5), 863-876 (2019).

Fong, M. y Loi, N., The Mediating Role of Self-compassion in Student Psychological Health, doi: 10.1111/ap.12185, Australian Psychologist, 51(6), 431-441 (2016).

Hone, L., Jarden A., Schofield G. y Duncan S., Measuring Flourishing: The impact of Operational Definitions on the Prevalence of High Levels of Wellbeing, doi: 10.5502/ijw.v4i1.4, International Journal of Wellbeing, 4(1), 62-90 (2014).

Howell, A. y Buro K., Measuring and Predicting Student Well-Being: Further Evidence in Support of Escala de Florecimiento (Diener et al., 2010) and the Scale of Positive and Negative Experiences, doi: 10.1007/s11205-014-0663-1, Social Indicators Research, 121(3), 903-915 (2015).

Huppert, F., Marks, N. y otros cinco autores, Measuring Well-being Across Europe: Description of the ESS Well-being Module and Preliminary Findings, doi: 10.1007/s11205-008-9346-0, Social Indicators Research, 91(3), $301-315$ (2009).

Jayawickreme, E., Forgeard, M. y Seligman, M., The Engine of Well-Being, doi: 10.1037/a0027990, Review of General Psychology, 16(4), 327-342 (2012).

Joshanloo, M., y Nosratabadi, M., Levels of Mental Health Continuum and Personality Rraits, doi: 10.1007/s11205-0089253-4, Social Indicators Research, 90(2), 211-224 (2009).

Jovanović, V., Structural validity of El Continuum de Salud Mental (Keyes, 2002): The Bifactor Model of Emotional, Social and Psychological Well-being, doi:10.1016/j.paid.2014.11.026, Personality and Individual Differences, 75, 154-159 (2015).

Keyes, C.L, Eisenberg, D. y otros cuatro autores, The Relationship of Level of Positive Mental Health With Current Mental Disorders in Predicting Suicidal Behavior and Academic Impairment in College Students, doi: 10.1080/07448481.2011.608393, Journal of American College Health, 60(2), 126-133 (2012).

Keyes, C.L., The Mental Health Continuum: From Languishing to Flourishing in Life, doi: 10.2307/3090197, Journal of Health and Social Behavior, 43(2), 207-222 (2002).

Knoesen, R., y Naudé, L., Experiences of Flourishing and Languishing during the First Year at University, doi: 10.1080/09638237.2017.1370635, Journal of Mental Health, 27(3), 269-278 (2018)

D'raven L.L., y Pasha-Zaidi, N., Using the PERMA Model in the United Arab Emirates, doi: 10.1007/s11205-015-0866-0, Social Indicators Research, 125(3), 905-933 (2015).

Lambert, L., Passmore, H. A., y Joshanloo, M., A Positive Psychology Intervention Program in a Culturally-Diverse University: Boosting happiness and reducing fear, doi: 10.1007/s10902-018-9993-z, Journal of Happiness Studies, 20(4), 1141-1162 (2019a).

Lambert, L., Passmore, H. A. y otros tres autores, Wellbeing matters in Kuwait: The Alnowair's Bareec Education Initiative, doi:10.1007/s11205-018-1987-z, Social Indicators Research, 143(2), 741-763 (2019b).

Low, K.G., Flourishing, Substance Use, and Engagement In Students entering College: A preliminary study, doi: 10.1080/07448481.2011.563432, Journal of American College Health, 59(6), 555-561 (2011).

Lloret-Segura, S., Ferreres-Traver, A., Hernández-Baeza A. y Tomás-Marco I., El Análisis Factorial Exploratorio de los ítems: una Guía Práctica, Revisada y Actualizada, doi: 10.6018/analesps.30.3.199361, Anales de Psicología, 30(3), 11511169 (2014).

Mesurado, B., Salanga, M. G., y Mateo, N.J., Basic Psychological Needs and Flourishing in Filipino University Students, doi: 10.1007/978-981-287-576-1_28, The Psychology of Asian Learners 459-469 (2016).

Miljković, D., y Jurčec, L., Is Curiosity Good for Students' Well-Being? The Case of the Faculty of Teacher Education and the Faculty of Kinesiology, doi: 10.15516/cje.v18i0.2184Hrvatski časopis za odgoj i obrazovanje, 18 (1), 103-121 (2016).

Moher, D., Liberati, A., Tetzlaff, J., y Altman, D.G., Preferred Reporting items for Systematic Reviews and Meta-Analyses: the PRISMA statement, Annals of Internal Medicine, ISSN: 15393704, 151(4), 264-269 (2009).

Muñiz, J., Elosua P. y Hambleton, R., Directrices para la traducción y adaptación de los Tests: segunda edición , doi: 10.7334/psicothema2013.24, Psicothema, 25(2), 151-157 (2013).

Negovan, V., Dimensions of Students' Psychosocial Well-being and their Measurement: Validation of a Students' Psychosocial Well Being Inventory, doi: 10.5964/ejop.v6i2.186, Europe's Journal of Psychology, 6(2), 85-104 (2010). 
Perestelo-Pérez, L., Standards on How to Develop and Report Systematic Reviews in Psychology and Health, doi: 10.1016/S1697-2600(13)70007-3, International Journal of Clinical and Health Psychology, 13(1), $49-57$ (2013).

Pozo-Muñoz,C., Garzón-Umerenkova, A., Bretones-Nieto, B. y Ligia-Charry, C., Psychometric Properties and Dimensionality of The flourishing Scale in Spanish-speaking population, doi: 10.14204/ejrep.38.15044, Electronic Journal of Research in Educational Psychology, 14(38), 175-192 (2016).

Ramírez-Maestre, C., Correa, M. y otros cuatro autores, Psychometric characteristics of The flourishing Scale -Spanish Version (FS-SV). The factorial Structure in two samples: Students and Patients with Chronic Pain, doi:10.1016/j.paid.2017.05.035, Personality and Individual Differences, 117, 30-36 (2017).

Rijavec, M., Ljubin-Golub, T., y Olčar, D., Can Learning for Exams Make Students Happy? Faculty-Related and FacultyUnrelated Flow Experiences and Well-Being, doi:10.15516/cje.v18i0.2223, Hrvatski časopis za odgoj i obrazovanje, 18(1), 153-164 (2016).

Robitschek, C. y Keyes, C. L., Keyes's Model of Mental Health with Personal Growth Initiative as a Parsimonious Predictor, doi: 10.1037/a0013954, Journal of Counseling Psychology, 56(2), 321-329 (2009).

Rose, T., Sean, J., y otros 4 autores, Measuring Mental Wellbeing Among Adolescents: A Systematic Review of Instruments, doi: 10.1007/s10826-017-0754-0, Journal of Child and Family Studies, 26(9), 2349-2362 (2017).

Rugira, J., Nienaber, A. y Wissing, M.P., Psychological Well-being among Tanzanian University Students, doi: 10.1080/14330237.2013.10820647, Journal of Psychology in Africa, 23(3), 425-429 (2013).

Sánchez -Meca, J., Cómo realizar una Revisión Sistemática y un Meta-Análisis , Aula Abierta, ISSN: 0210-2773, 38(2), 53-64 (2010).

Satici, S. y Uysal, R., Well-being and Problematic Facebook Use, doi:10.1016/j.chb.2015.03.005, Computers in Human Behavior, 49, 185-190 (2015).

Satici, S., Uysal, R. y Akin, A., Investigating the Relationship Between Flourishing and Self-Compassion: a Structural Equation Modeling Approach, doi:10.5334/pb-53-4-85, Psychologica Belgica, 53(4) 85-99 (2013).

Selvaraj, P.R., y Bhat, C.S., Predicting the Mental Health of College Students with Psychological Capital, doi: 10.1080/09638237.2018.1469738, Journal of Mental Health, 27(3), 279-287 (2018)

Seyranian, V., Madva, A. y otros cuatro autores, The longitudinal effects of STEM identity and gender on flourishing and achievement in college physics, doi: 10.1186/s40594-018-0137-0, International journal of STEM education 5 (49) 1-14 (2018).

Schotanus-Dijkstra, M.E., Pieterse, M. y otros seis autores, What factors are associated with Flourishing? Results from a Large Representative National Sample, doi:10902-015-9647-3, Journal of Happiness Studies, 17(4), 1351-1370 (2016a).

Schotanus-Dijkstra, M.E, Ten Have, M. y otros tres autores, The Longitudinal Relationship between Flourishing Mental Health And Incident Mood, Anxiety bnd Substance Use Disorders, doi: 10.1093/eurpub/ckw202, The European Journal of Public Health, 27(3) 563-568 (2016b).

Seligman, M. E., Flourish: A visionary new understanding of Happiness and Well-Being. 1era edición, 10-344. Free Press, New York, Estados Unidos (2011)

Shellman, A. y Hill E., Flourishing through Resilience: The Impact of a College Outdoor Education Program, doi: 10.18666/JPRA-2017-V35-I4-7779, Journal of Park and Recreation Administration, 35(4), 59-68 (2017).

Singh, K., Bassi M., Junnarkar M. y Negri L., Mental Health and Psychosocial Functioning in Adolescence: an investigation among Indian Students from Delhi, doi: 10.1016/j.adolescence.2014.12.008, Journal of Adolescence, 39, 59-69 (2015).

Stallman, H., Psychological Distress in University Students: a comparison with General Population Data, doi:10.1080/00050067.2010.482109, Australian Psychologist, 45(4), 249-257 (2010).

Stoeber, J., y Corr, P., A short empirical note on Perfectionism and Flourishing, doi:10.1016/j.paid.2015.10.036, Personality and Individual Differences, 90, 50-53 (2016).

Streiner, D., Norman, G. y Cairney, J., Health Measurement Scales: a Practical Guide to their Development and use, Oxford University Press, Oxford ,Canadá (2015).

Sumi, K., Reliability and Validity of Japanese Versions of The flourishing Scale and the Scale of Positive and Negative Experience, doi:10.1007/s11205-013-0432-6, Social Indicators Research, 118(2), 601-615 (2014).

Uysal, R., The Predictive Roles of Social Safeness and Flourishing on Problematic Facebook Use, doi: 10.1177/0081246314560010, South African Journal of Psychology, 45(2), 182-193 (2015a).

Uysal, R., Social Competence and Psychological Vulnerability: The mediating role of Flourishing, doi: 10.2466/21.PR0.117c18z2, Psychological Reports, 117(2), 554-565 (2015b).

Van Zyl, Ll. y Rothmann, S., Flourishing of Students in a Tertiary Education Institution in South Africa, doi: 10.1080/14330237.2012.10820573, Journal of Psychology in Africa, 22(4), 593-599 (2012).

Venning, A., Wilson, A., Kettler, L. y Eliott, J., Mental Health among youth in South Australia: A survey of Flourishing, Languishing, Struggling, And Floundering, doi:10.1111/j.1742-9544.2012.00068.x, Australian Psychologist, 48(4), 299-310 (2013). 
Villieux, A., Sovet, L., Jung, S.C. y Guilbert, L., Psychological Flourishing: Validation of the French Version of The Flourishing Scale and Exploration of its relationships with Personality Traits, doi: 10.1016/j.paid.2015.08.027, Personality and Individual Differences, 88, 1-5 (2016).

Vorone, S., Vorobyov, A. y Negovan, V., Are dimensions of Psycho-Social Well-Being different among Latvian And Romanian University Students?, doi:10.1016/j.sbspro.2012.01.243, Procedia-Social and Behavioral Sciences, 33, 855859 (2012).

Waterman, A., Reconsidering Happiness: a Eudaimonist's Perspective, doi: 10.1080/17439760802303002, The Journal of Positive Psychology, 3(4), 234-252 (2008).

Witten, H., Savahl S. y Adams S., Adolescent flourishing: A systematic review, doi: 10.1080/23311908.2019.1640341, Cogent Psychology, 6, 1-16 (2019).

Wolbert, L., De Ruyter D. y Schinkel A., Formal criteria for the concept of Human Flourishing: the first step in Defending Flourishing as an ideal aim of Education, doi:10.1080/17449642.2014.998032, Ethics and Education, 10(1), 118-129 (2015). 
